\title{
1 Transcriptome atlas of Phalaenopsis equestris
}

Anna V. Klepikova ${ }^{1}$, Artem S. Kasianov ${ }^{1}$, Margarita A. Ezhova ${ }^{1}$, Aleksey A. Penin ${ }^{1,2}$, Maria D. Logacheva ${ }^{1,2,3}$

${ }^{1}$ Laboratory of Plant Genomics, Institute for Information Transmission Problems of the Russian Academy of Sciences, Moscow, Russia

${ }^{2}$ Faculty of Biology, Lomonosov Moscow State University, Moscow, Russia

${ }^{3}$ Center of Life Sciences, Skolkovo Institute of Science and Technology, Moscow, Russia

Corresponding Author:

Anna V. Klepikova ${ }^{1}$

Bolshoy Karetny per. 19, build. 1, Moscow 127051, Russia

Email address: annklepikova@gmail.com

\section{Abstract}

The vast diversity of Orchidaceae together with sophisticated adaptations to pollinators and other unique features make this family an attractive model for evolutionary and functional studies. The sequenced genome of Phalaenopsis equestris facilitates Orchidaceae research. Here we present an RNA-seq based transcriptome map of $P$. equestris which covers 19 organs of the plant including leaves, roots, floral organs and shoot apical meristem. We demonstrated the high quality of the data and showed the similarity of $P$. equestris transcriptome map with gene expression atlases of other plants. The transcriptome map can be easily accessed through our database Transcriptome Variation Analysis (TraVA) visualizing gene expression profiles. As an example of the application we analyzed the expression of Phalaenopsis "orphan" genes - the ones that do not have recognizable similarity with genes of other plants. We found that about a half of them are not expressed; the ones that are expressed have a predominant expression pattern in reproductive structures.

\section{Introduction}

The enormous diversity of orchids traditionally attracts attention of plant biologists. Orchidaceae comprises about 25 thousand of species, which makes it the largest plant taxon (Cai et al., 2015). The diversification of orchids has evolved along with complex pollinator-adapted flower structure (Cozzolino \& Widmer, 2005), CAM-photosynthesis and epiphytism (Silvera et al., 2009).

Genome assembly of Phalaenopsis equestris (the horse phalaenopsis) (Cai et al., 2015) provided novel opportunities for evolutionary and functional studies of Orchidaceae. Genome assembly was used for the functional studies of transcription factor families (Lin et al., 2016; Valoroso et al., 2019), somatic embryogenesis (Chen et al., 2019), retrotransposon insertions (Hsu et al., 2019), as well as for evolutionary studies of ancient polyploidy (Barrett et al., 2019). However, 
transcriptome resources of $P$. equestris remain limited even though the de novo transcriptome assembly was performed based on RNA sequencing of 11 organs (Niu et al., 2016). In our study we present a transcriptome map of $P$. equestris consisting of 19 samples in two biological replicates. High-quality RNA of orchid organs and tissues was sequenced using Illumina technology resulting in $1687 \mathrm{M}$ reads. We compared expression characteristics of $P$. equestris transcriptome map with gene expression atlases of other plants to provide evidence of reliability of our data. Transcriptome map of $P$. equestris can be applied in a great variety of functional studies.

\section{Materials \& Methods}

\section{Growing conditions}

Plants were grown in a climate chamber under a $16 \mathrm{~h}$ light $/ 8 \mathrm{~h}$ dark cycle at $22^{\circ} \mathrm{C}$ and $50-60 \%$ relative humidity. Samples were collected in two biological replicates; each replicate consists of to reduce the influence of the circadian cycle.

\section{RNA extraction, library preparation and sequencing}

RNA was extracted using the RNeasy mini kit (Qiagen, The Netherlands) following the (New England BioLabs, MA, USA) following the manufacturer's protocol in 0.5 of the recommended volume (due to low RNA quantity in such samples as shoot apical meristem). cDNA libraries were sequenced with the HiSeq4000 and NextSeq500 (Illumina, CA, USA) instruments (50 bp and 75 bp single read run).

\section{Read mapping}

66 Read trimming was performed using Trimmomatic version 0.36 (Bolger, Lohse \& Usadel, 2014) in a single read mode and parameters "ILLUMINACLIP:common.adapters.file:2:30:10 LEADING:20 TRAILING:20 SLIDINGWINDOW:4:15 MINLEN:30". For read mapping genome assembly and annotation of $P$. equestris from PLAZA database (version 4.5) was used. Trimmed reads mapped on the genome assembly using Spliced Transcripts Alignment to a Reference (STAR) version 2.4.2 (Dobin et al., 2013) in the "GeneCounts" mode and parameters “--sjdbOverhang 59 --sjdbGTFfeatureExon exon --sjdbGTFtagExonParentTranscript gene_id" to obtain counts of uniquely mapped reads on each gene.

75 Gene read counts obtained with STAR were normalized on library size using size factors, as described in (Anders \& Huber, 2010). A threshold of five or higher normalized read counts in each biological replicate was used to define expressed genes.

To describe gene expression pattern Shannon entropy values were calculated for expressed in at least one sample genes (Schug et al., 2005). In order to avoid overrepresentation of certain plant 
averaged if samples had distance $\left(1-\right.$ Pearson $\left.r^{2}\right)$ less than 0.1 . Sample groups are listed in Table S1.

\section{Data availability}

The RNA-seq raw data of transcriptome map were deposited in NCBI Sequence Read Archive (SRA) under BioProject accession PRJNA667255. The TraVA database can be accessed at http://travadb.org/browse/Species=Phalaenopsis_equestris/.

\section{Results}

\section{Transcriptome map construction}

Ornamental orchid $P$. equestris comprises three varieties and numerous hybrids of various flower colors and sizes (Hsu \& Chen, 2016). To create transcriptome atlas we chose P. equestris var. blue (orchidee.su) as clonal plants are available for the cultivar which helps to reduce interindividual variability. We have collected 31 samples covering main plant organs and developmental stages such as roots, young and mature leaves, floral organs, flower buds, and meristems. Each sample was collected in two biological replicates, and each replicate was pooled from at least seven plants. Sample RNA was sequenced on Illumina platform resulting in $29 \mathrm{M}$ $65 \mathrm{M}$ raw single reads (38 M median) for each sample (for sequencing statistics see Table S2). After removing low-quality reads and technical sequenced $98.7-99.8 \%$ of reads remained (Table S2).

Reads were mapped on the reference genome of $P$. equestris (Cai et al., 2015) with only one match allowed (unique mapping); 9.2-89.6\% of high-quality reads were successfully mapped (Table S2). 12 samples showed extremely low percentage of read mapping; unmapped reads were identified as sequences belonging to Cymbidium mosaic virus (GenBank accession MK816927) which are known to persist in the majority of $P$. equestris population and affect mainly mature and senescent tissues (Koh, Lu \& Chan, 2014). As the library size of infected samples was insufficient and can distort the conclusions we excluded samples with a percentage of mapped read lower than $35 \%$ in at least one biological replicate. The remained samples had $37.3-89.6 \%$ of uniquely mapped reads with median of $81.6 \%$. Thus, we constructed a transcriptome map of $P$. equestris covering 19 organs and parts of the plant. Floral organs (anthers, labellum, inner and outer tepals), leaves at different developmental stages, axes (inflorescence and pedicel), shoot apical and inflorescence meristems, and root parts were taken into analysis (for detailed description of samples see Table S3). The biological replicates showed high consistency (median Pearson $\mathrm{r}^{2}=0.99$, Table S4). Clustering of samples generally reflects plant body plan and groups organs with similar morphology and physiology (Klepikova \& Penin, 2019). Hierarchical clustering of P. equestris samples showed the same pattern (Fig. 1A). Sample clusters were formed by floral organs, leaf parts, meristems and young leaves, inflorescence internode and root; young and mature anthers were an outgroup for the other samples, similar to A. thaliana, rice, and maize (Nobuta et al., 2007; Wang et al., 2010; Stelpflug et al., 2016; Klepikova et al., 2016). The distances between samples on clustering tree were closer than in other species we observed (Klepikova et al., 2016; 
Penin et al., 2019), which can be explained by the lack of older tissues in P. equestris

transcriptome atlas.
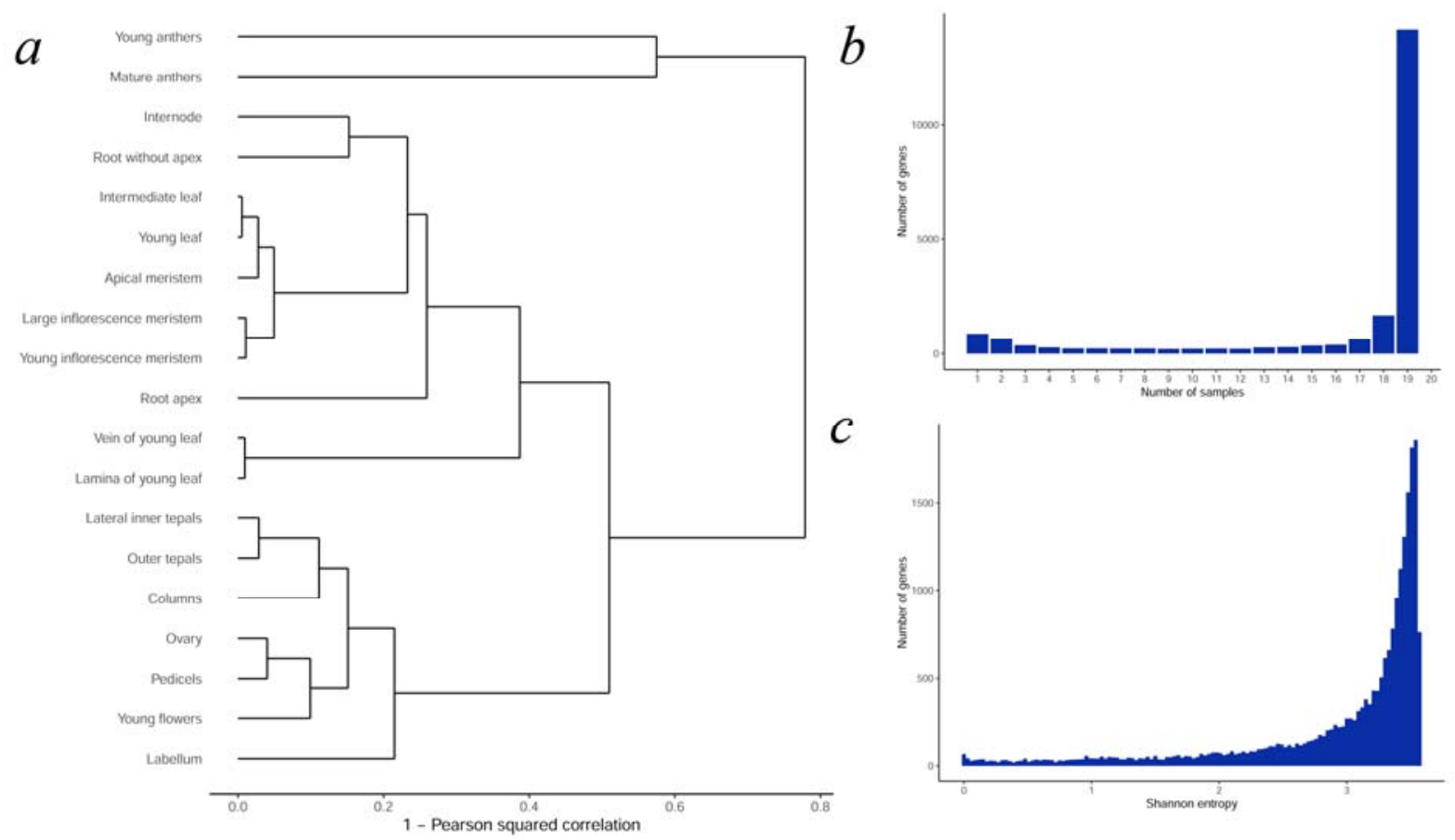

Figure 1. Expression characteristics of the P. equestris transcriptome map: (a) Hierarchical clustering tree of transcriptome map samples; (b) The distribution of genes by the number of samples where gene is expressed. Only expressed genes with 5 or more normalized read counts in each biological replicate were considered; (c) The distribution of Shannon entropy of P. equestris genes. We compare our samples with publicly available $P$. equestris transcriptomes (Table S5). In general, the clustering of samples was consistent (Fig. S1), though leaf and column from the BioProject PRJNA288388 (Niu et al., 2016) form outgroup to all other samples.

Phalaenopsis genome annotation (PLAZA database, version 4.5) includes 29431 protein-coding genes. Among them 14174 (48\%) genes were expressed in all samples (using five reads in each biological replicate as a threshold), when transcripts of 21671 (74\%) genes were found in at least one sample. These values are in the range of typical expressed gene numbers across plant transcriptome maps (Klepikova \& Penin, 2019). As in other species, samples demonstrated similarity in the number of expressed genes, which varied form 15612 (53\%) in shoot apical meristem to 18947 (64\%) in ovules before pollination (Table S6).

\section{Expression patterns of $P$. equestris genes}

The study of gene expression pattern can shed light on the biological function of the gene and place it among essential for a plant existence ubiquitously expressed genes or precise regulators of tissue features - sample-specific expressed genes. We used two approaches to define gene expression patterns. A number of samples where gene is expressed is the simplest method to 
145 characterize expression pattern width, as was shown for Nicotiana tabacum (Edwards et al.,

146 2010) or Vigna unguiculata (Yao et al., 2016). The majority of genes (16 486) were expressed in

14717 or more samples; the second peak (1 896 genes) of the distribution is formed by genes

148 expressed in 3 or less samples (Fig. 1B). The main patterns of tissue-specific genes were anthers

149 (56\% of tissue-specific genes), roots (11\%), and meristems (both shoot apical and inflorescence

150 meristem, 8\%). The high number of anther-associated genes are known for A. thaliana

151 (Klepikova et al., 2016) and is expected for P. equestris as young and mature anthers are the

152 most distant samples on clustering tree (Fig. 1A).

153 While useful, such approach depends on an arbitrary threshold which separates expressed and

154 non-expressed genes and does not take into account the variation of expression level between

155 samples. To overcome the issue, we used Shannon entropy as a measure of expression pattern

156 width: low entropy values correspond to tissue-specific genes, while high values mark

157 ubiquitously expressed genes (Schug et al., 2005). The distribution of Shannon entropy in

$158 P$. equestris was significantly skewed to the right revealing major part of wide-expressed genes

159 (Fig. 1C) similarly to A. thaliana, Solanum licopersicum and Zea mays (Sekhon et al., 2013;

160 Klepikova et al., 2016; Penin et al., 2019).

161 Using Shannon entropy value lower than 0.25 we identified 521 tissue-specific genes. As in case

162 of direct count the majority of genes was associated with anthers or roots (Fig. 2). According to

163 GO enrichment, genes uniquely expressed in the mature anthers were involved in cell wall

164 organization, biogenesis and modification and had pectinesterase and enzyme inhibitor activity

165 (Table S7). Young anthers were characterized by genes encoding products with amine and amino

166 acid binding activity (Table S8). Root-specific genes (expressed in the sample "Root without

167 apex") were described by terms "response to chemical stimulus", "response to oxidative stress",

168 “oxidation reduction", and "heme binding" (Table S9). 


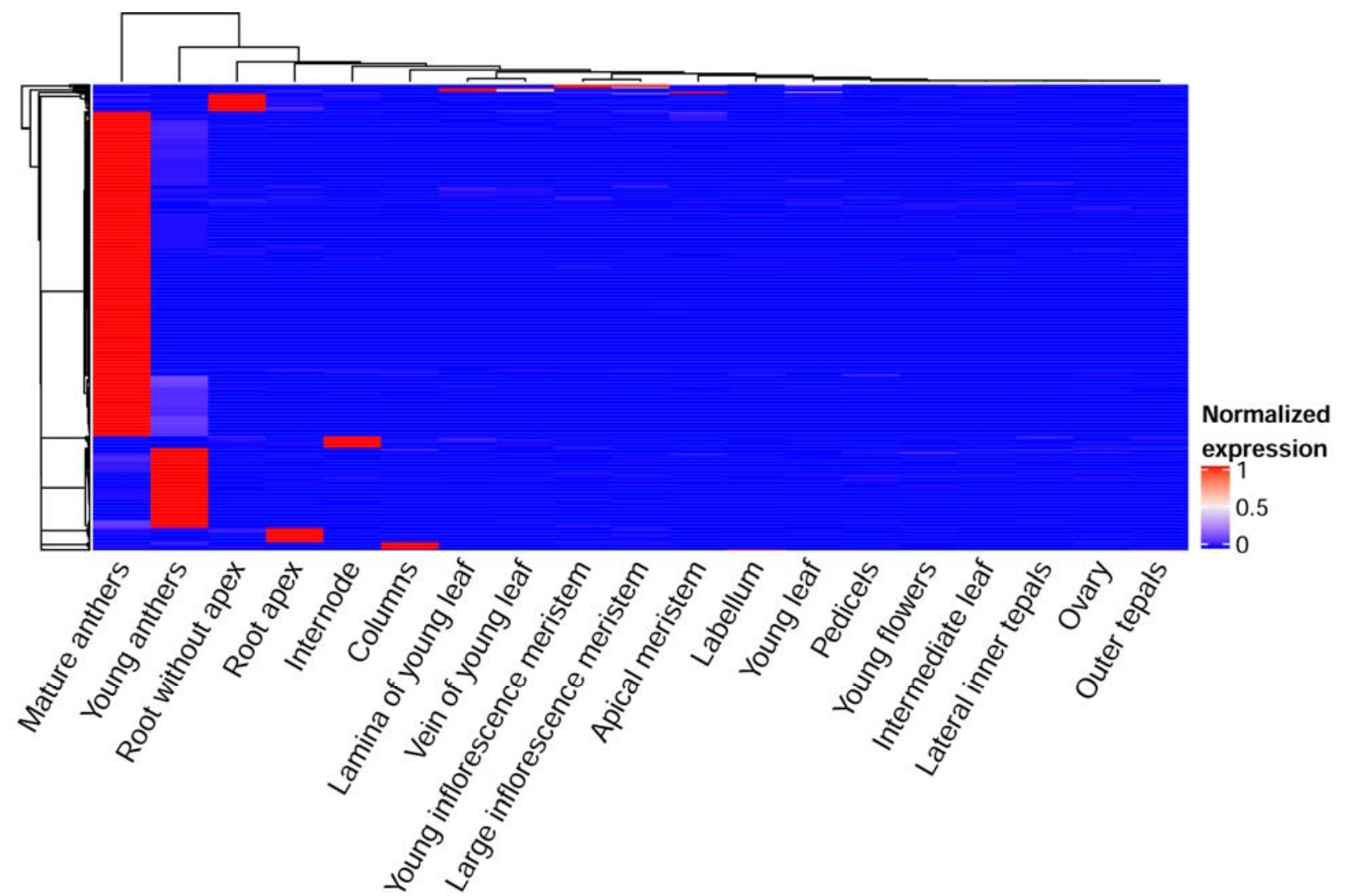

Figure 2. The heatmap of tissue-specific genes. Expression levels of each gene in each sample were normalized on its maximal expression level.

To find genes with opposite behaviour which uniformly expressed across tissues we selected genes with Shannon entropy 3.55 or higher and calculated coefficient of variance $(\mathrm{CV})$ as a measure of expression stability. For 899 out of 1340 genes CV was less than 0.25 , indicating uniform expression in all samples and biological replicates. Stable genes had GO enrichment in terms associated with vesicles, membranes, RNA processing and localization. The list of GO categories strongly overlapped with the enrichment of $A$. thaliana uniformly expressed genes indicating inter-species universality of basic biological processes (Table S10).

180 We aimed to make our transcriptome data easily accessible and ready to use, so we uploaded $P$. equestris transcriptomes into our database Transcriptome Variation Analysis (TraVA, http://travadb.org/browse/Species=Phalaenopsis_equestris/). TraVA interface demonstrates a color chart of gene expression profiles in a single- or multiple-gene view. A user can prefer to show or hide expression values in a chart and choose between several types of read count normalization (Fig. 3). 


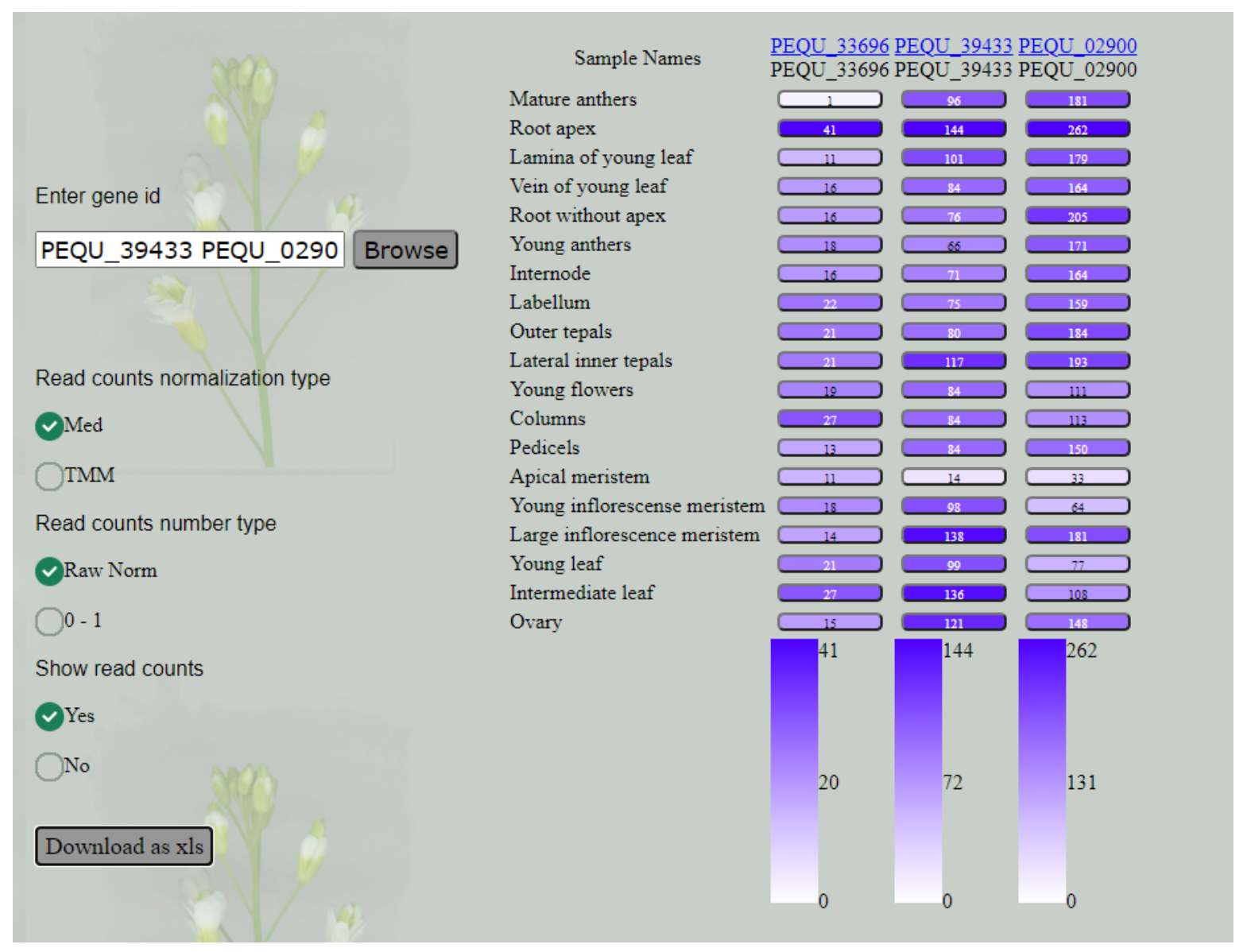

Figure 3. Database view.

Graphical interface of TraVA facilitate gene expression patterns analysis and comparison and can be widely used in P. equestris functional studies. Orchids are a large and highly diverse plant family whose species adapted to a number of ecological niches (typical terrestrial plants, epiphytes, non-photosynthetic plants). These adaptations reflect in their genome - for example, P. equestris which has sophisticatedly differentiated perianth the number of AP3 orthologs is higher compared to Apostasia schenzhenica, the basal orchid species with undifferentiated perianth. Vice versa, $P$. equestris which is an epiphyte and does not develop typical terrestrial roots, lacks AGL12 and several genes of the ANRI clade, in contrast to A. schenzhenica. This stresses the importance of the study of lineage-specific genes and gene families. For the genes that do not have orthologs in model species the analysis of the expression profiles is the first step towards functional characterization. cut-off $=10$ ). Out of them 118 share similarity with the proteins of $A$. schenzhenica and are thus presumably orchid-specific while 42 have no hits and thus emerged after the divergence of 

available under aCC-BY-NC 4.0 International license.

204 Apostasioideae and Epidendroideae. The survey of the expression profiles showed that 93 of 205 them are not expressed in any of the samples of the map (Fig. S2). Among the ones which are 206 expressed most are expressed at very low levels. Higher expression levels are associated with 207 reproductive structures, in particular, anthers (Fig. 4).

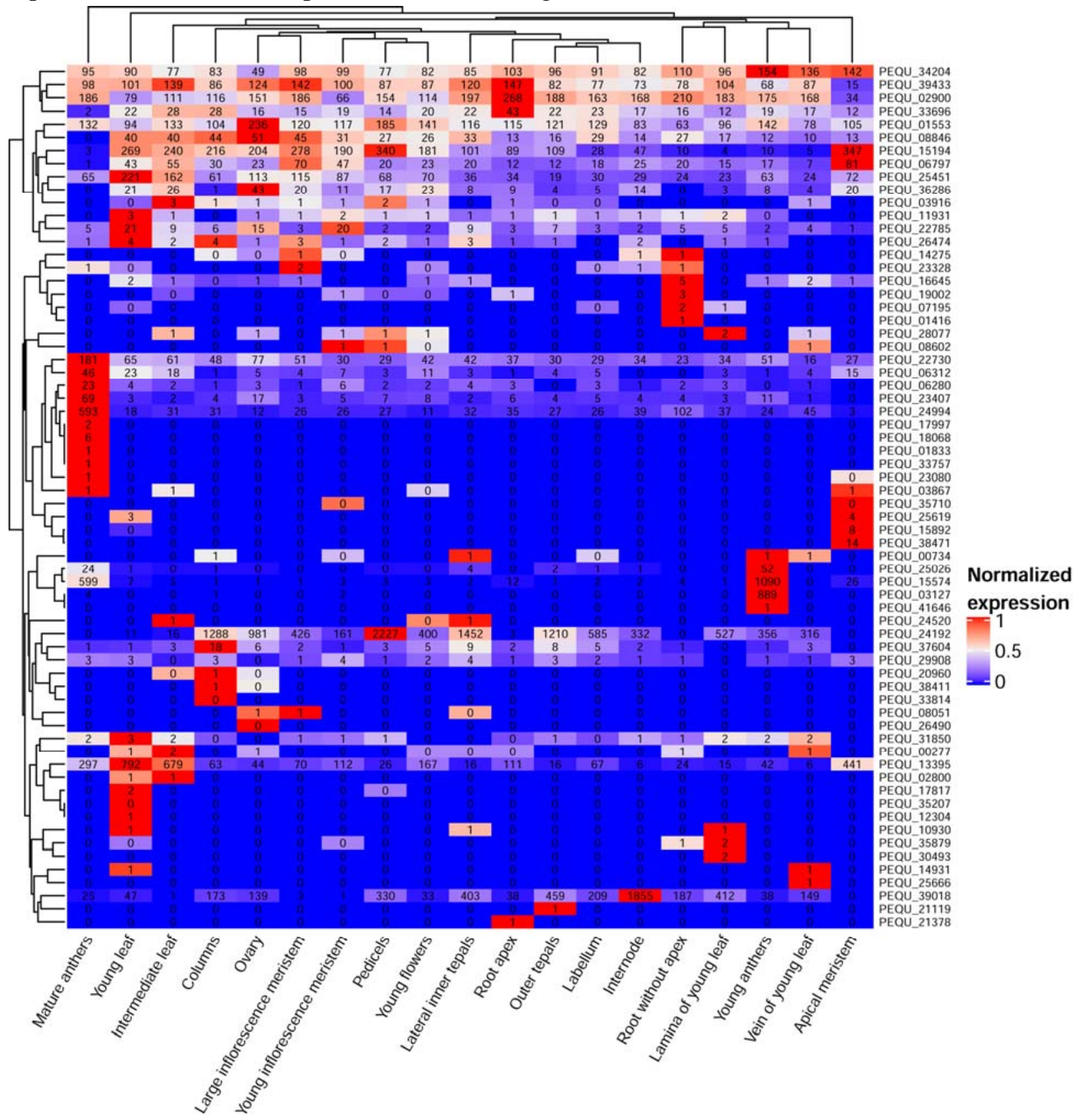

Figure 4. The heatmap of P. equestris-specific genes. Expression levels of each gene in each sample were normalized on its maximal expression level for the color key. The numbers on the

211 figure represent normalized gene read count averaged over biological replicates.

212 Among vegetative structures the most distinct is root (root apex) where three genes -

213 PEQU_39433, PEQU_02900,PEQU_33696 - have the highest expression levels. Phalaenopsis

214 roots are unique (compared to most other plants, including $A$. schenzhenica, but not to other 
epiphytic orchids) in many respects - in particular, they are photosynthetic and develop a special structure called velamen. Velamen is a tissue of epidermal origin that consists of several layers of dead cells which help to absorb water and protect photosynthetic tissues of the root from the UV damage. Notably, PEQU_39433 and PEQU_33696 do not have homologs in A. schenzhenica. PEQU_02900 has a marginal similarity (34\%) with A. schenzhenica protein encoded by Ash001570 gene. widely discussed, in particular in application to plants (Arendsee, Li \& Wurtele, 2014). While a part of the orphan genes might represent the artifacts of the annotation, others have a function (for example, A. thaliana orphan gene $Q Q S$ which acts in starch metabolism (Li et al., 2009). The functional analysis of orphan genes however lags behind the typical genes; they are overlooked in the annotations based on the homology; they are also usually expressed at lower levels and in a narrower range of tissues (reviewed in Schlötterer, 2015). The study of expression levels and patterns of a potential orphan gene is a first step towards its characterization - the detectable level of expression is an evidence of that the ORF is indeed a gene, not an annotation artifact.

Notably that orphan genes in the well-characterized animal objects (Drosophila, primates) have expression patterns biased towards male reproductive structures (Begun et al., 2007; Xie et al., 2012). According to the "out-of-testis" hypothesis (Kaessmann, 2010) this is mediated by the unique epigenetic state of the chromatin during male gametogenesis. We observed the same bias in Phalaenopsis; the growing availability of plant transcriptome maps will enable to find out if this is universal for plants.

\section{Conclusions}

In this study we present a transcriptome map of orchid Phalaenopsis equestris covering 19 organs at various stages of the development. We identified 521 tissue-specific genes the majority of which expressed in anthers, roots (11\%), and meristems. The uniformly expressed genes were associated with the similar processes as in Arabidopsis thaliana, i.e. vesicles, membranes, RNA processing and localization. In order to improve reuse of the data we integrated transcriptome

\section{Author Contributions} acquisition, A.A.P.; Investigation, A.A.P., M.D.L. and A.V.K.; Methodology, A.A.P and M.A.E.; Project administration, A.A.P.; Software, A.V.K. and A.S.K.; Supervision, A.A.P.;

\section{Funding}

252 The reported study was funded by RFBR according to the research project № 18-29-13017.

\section{Conflicts of Interest}

254 The authors declare no conflict of interest. 


\section{References}

Anders S, Huber W. 2010. Differential expression analysis for sequence count data. Genome Biology 11:R106. DOI: 10.1186/gb-2010-11-10-r106.

Arendsee ZW, Li L, Wurtele ES. 2014. Coming of age: orphan genes in plants. Trends in Plant Science 19:698-708. DOI: 10.1016/j.tplants.2014.07.003.

Barrett CF, McKain MR, Sinn BT, Ge X-J, Zhang Y, Antonelli A, Bacon CD. 2019. Ancient Polyploidy and Genome Evolution in Palms. Genome Biology and Evolution 11:1501-

Begun DJ, Lindfors HA, Kern AD, Jones CD. 2007. Evidence for de Novo Evolution of Testis-

Expressed Genes in the Drosophila yakuba / Drosophila erecta Clade. Genetics 176:11311137. DOI: 10.1534/genetics.106.069245.

Bolger AM, Lohse M, Usadel B. 2014. Trimmomatic: a flexible trimmer for Illumina sequence data. Bioinformatics (Oxford, England) 30:2114-2120. DOI:

10.1093/bioinformatics/btu170.

Cai J, Liu X, Vanneste K, Proost S, Tsai W-C, Liu K-W, Chen L-J, He Y, Xu Q, Bian C, Zheng Z, Sun F, Liu W, Hsiao Y-Y, Pan Z-J, Hsu C-C, Yang Y-P, Hsu Y-C, Chuang Y-C, Dievart A, Dufayard J-F, Xu X, Wang J-Y, Wang J, Xiao X-J, Zhao X-M, Du R, Zhang GQ, Wang M, Su Y-Y, Xie G-C, Liu G-H, Li L-Q, Huang L-Q, Luo Y-B, Chen H-H, Van de Peer Y, Liu Z-J. 2015. The genome sequence of the orchid Phalaenopsis equestris. Nature Genetics 47:65-72. DOI: 10.1038/ng.3149.

Chen J-C, Tong C-G, Lin H-Y, Fang S-C. 2019. Phalaenopsis LEAFY COTYLEDON1-Induced Somatic Embryonic Structures Are Morphologically Distinct From Protocorm-Like Bodies. Frontiers in Plant Science 10:1594. DOI: 10.3389/fpls.2019.01594.

Cozzolino S, Widmer A. 2005. Orchid diversity: an evolutionary consequence of deception? Trends in Ecology \& Evolution 20:487-494. DOI: 10.1016/j.tree.2005.06.004.

Dobin A, Davis CA, Schlesinger F, Drenkow J, Zaleski C, Jha S, Batut P, Chaisson M, Gingeras TR. 2013. STAR: ultrafast universal RNA-seq aligner. Bioinformatics (Oxford, England) 29:15-21. DOI: 10.1093/bioinformatics/bts635.

Edwards KD, Bombarely A, Story GW, Allen F, Mueller LA, Coates SA, Jones L. 2010. TobEA: an atlas of tobacco gene expression from seed to senescence. BMC Genomics 11:142. DOI: 10.1186/1471-2164-11-142.

Hsu C-C, Chen W-H. 2016. The breeding achievements from Phalaenopsis equestris. In: Malayan Orchid Review. WORLD SCIENTIFIC, 41-47. DOI: 10.1142/9789814749954_0007.

Hsu C-C, Su C-J, Jeng M-F, Chen W-H, Chen H-H. 2019. A HORT1 Retrotransposon Insertion in the PeMYB11 Promoter Causes Harlequin/Black Flowers in Phalaenopsis Orchids. Plant Physiology 180:1535-1548. DOI: 10.1104/pp.19.00205.

Kaessmann H. 2010. Origins, evolution, and phenotypic impact of new genes. Genome Research 20:1313-1326. DOI: 10.1101/gr.101386.109. 
Klepikova AV, Kasianov AS, Gerasimov ES, Logacheva MD, Penin AA. 2016. A High Resolution Map of the Arabidopsis thaliana Developmental Transcriptome Based on RNA-seq Profiling. The Plant Journal. DOI: 10.1111/tpj.13312.

Klepikova AV, Penin AA. 2019. Gene Expression Maps in Plants: Current State and Prospects. Plants 8:309. DOI: 10.3390/plants8090309.

Koh KW, Lu H-C, Chan M-T. 2014. Virus resistance in orchids. Plant Science 228:26-38. DOI: 10.1016/j.plantsci.2014.04.015.

Li L, Foster CM, Gan Q, Nettleton D, James MG, Myers AM, Wurtele ES. 2009. Identification of the novel protein QQS as a component of the starch metabolic network in Arabidopsis leaves. The Plant Journal 58:485-498. DOI: 10.1111/j.1365-313X.2009.03793.x.

Lin Y-F, Chen Y-Y, Hsiao Y-Y, Shen C-Y, Hsu J-L, Yeh C-M, Mitsuda N, Ohme-Takagi M, Liu Z-J, Tsai W-C. 2016. Genome-wide identification and characterization of TCP genes involved in ovule development of Phalaenopsis equestris. Journal of Experimental Botany 67:5051-5066. DOI: 10.1093/jxb/erw273.

Niu S-C, Xu Q, Zhang G-Q, Zhang Y-Q, Tsai W-C, Hsu J-L, Liang C-K, Luo Y-B, Liu Z-J. 2016. De novo transcriptome assembly databases for the butterfly orchid Phalaenopsis equestris. Scientific Data 3:160083. DOI: 10.1038/sdata.2016.83.

Nobuta K, Venu RC, Lu C, Beló A, Vemaraju K, Kulkarni K, Wang W, Pillay M, Green PJ, Wang G, Meyers BC. 2007. An expression atlas of rice mRNAs and small RNAs. Nature Biotechnology 25:473-477. DOI: 10.1038/nbt1291.

Penin AA, Klepikova AV, Kasianov AS, Gerasimov ES, Logacheva MD. 2019. Comparative Analysis of Developmental Transcriptome Maps of Arabidopsis thaliana and Solanum lycopersicum. Genes 10:50. DOI: 10.3390/genes10010050.

Schlötterer C. 2015. Genes from scratch - the evolutionary fate of de novo genes. Trends in Genetics 31:215-219. DOI: 10.1016/j.tig.2015.02.007.

Schug J, Schuller W-P, Kappen C, Salbaum JM, Bucan M, Stoeckert CJ. 2005. Promoter features related to tissue specificity as measured by Shannon entropy. Genome Biology 6:R33. DOI: 10.1186/gb-2005-6-4-r33.

Sekhon RS, Briskine R, Hirsch CN, Myers CL, Springer NM, Buell CR, de Leon N, Kaeppler SM. 2013. Maize Gene Atlas Developed by RNA Sequencing and Comparative Evaluation of Transcriptomes Based on RNA Sequencing and Microarrays. PLoS ONE 8:e61005. DOI: 10.1371/journal.pone.0061005.

Silvera K, Santiago LS, Cushman JC, Winter K. 2009. Crassulacean Acid Metabolism and Epiphytism Linked to Adaptive Radiations in the Orchidaceae. Plant Physiology 149:1838-1847. DOI: 10.1104/pp.108.132555.

Stelpflug SC, Sekhon RS, Vaillancourt B, Hirsch CN, Buell CR, de Leon N, Kaeppler SM. 2016. An Expanded Maize Gene Expression Atlas based on RNA Sequencing and its Use to Explore Root Development. The Plant Genome 9:0. DOI: 10.3835/plantgenome2015.04.0025. 
334 Valoroso MC, Sobral R, Saccone G, Salvemini M, Costa MMR, Aceto S. 2019. Evolutionary 335 Conservation of the Orchid MYB Transcription Factors DIV, RAD, and DRIF. Frontiers $336 \quad$ in Plant Science 10:1359. DOI: 10.3389/fpls.2019.01359.

337 Wang L, Xie W, Chen Y, Tang W, Yang J, Ye R, Liu L, Lin Y, Xu C, Xiao J, Zhang Q. 2010. A 338 dynamic gene expression atlas covering the entire life cycle of rice. The Plant Journal 61:752-766. DOI: 10.1111/j.1365-313X.2009.04100.x.

340 Xie C, Zhang YE, Chen J-Y, Liu C-J, Zhou W-Z, Li Y, Zhang M, Zhang R, Wei L, Li C-Y. 341 2012. Hominoid-Specific De Novo Protein-Coding Genes Originating from Long Non342 Coding RNAs. PLoS Genetics 8:e1002942. DOI: 10.1371/journal.pgen.1002942.

343 Yao S, Jiang C, Huang Z, Torres-Jerez I, Chang J, Zhang H, Udvardi M, Liu R, Verdier J. 2016.

$344 \quad$ The Vigna unguiculata Gene Expression Atlas (VuGEA) from de novo assembly and 345 quantification of RNA-seq data provides insights into seed maturation mechanisms. The $346 \quad$ Plant Journal 88:318-327. DOI: 10.1111/tpj.13279. 